\title{
Formins initiate new actin filaments
}

Good news - a new mechanism for nucleating actin filaments has been discovered. Two recent studies (Pruyne, D. et al. Science, June 6, 2002 (10.1126/science.1072309) and Sagot, I. et al. Nature Cell Biology, 4, 616-621, (2002)) have shown that formins stimulate the assembly of filaments from actin monomers. Their biochemical studies with fragments of budding yeast formins, as well as genetic and cellular analyses, support the hypothesis that formins promote the assembly of bundles of actin filaments (see Figure).

Proteins that initiate the formation of actin filaments have remained elusive. The best-characterized initiator of actin filaments, the Arp $2 / 3$ complex, was discovered 25 years after actin was identified in non-muscle cells. Proteins that cap the fastgrowing barbed end of actin filaments initiate polymerization, but growth is thought to proceed in the opposite direction to most cellular filaments.

Formins differ in many ways from Arp2/3 complex: they are large, multi-domain polypeptides with relative molecular masses in excess of $200 \mathrm{~K}$, whereas the $225 \mathrm{~K}$ Arp $2 / 3$ complex consists of two actin-related proteins (Arps), a WD40 $\beta$ propeller and four subunits with novel folds. Rho family GTPases activate auto-inhibited formins directly, whereas they activate the Arp $2 / 3$ complex indirectly by reversing the auto-inhibition of WASp family proteins. Active WASp stimulates Arp2/3 complex by stabilizing an active conformation. Formins produce unbranched filaments, whereas the Arp $2 / 3$ complex initiates a new filament as a $70^{\circ}$ branch on the side of a pre-existing filament. The Arp $2 / 3$ complex caps the slow-growing pointed end of the new actin filament, leaving the fast-growing barbed end to elongate. Formins seem to localize near barbed ends, but both these studies argue that the new filaments grow from the barbed ends. The Arp $2 / 3$

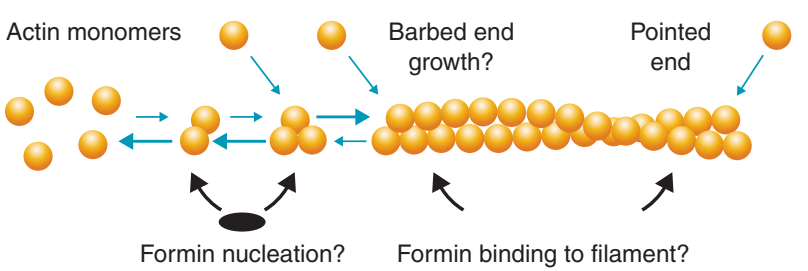

complex is much more potent in promoting filament formation than the formins used in these two studies. Each Arp2/3 complex produces a filament under optimal conditions, whereas the ratio of new filaments to formins is less than 0.02 , assuming that filaments grow from their barbed ends. A calculation based on partial capping of barbed ends gives more new filaments per formin.

This exciting new work on formins raises many questions about their mechanism of actin filament nucleation. Why is nucleation so inefficient? Are full-length formins activated by GTPases more effective than the fragments used in these studies? Do the new filaments really grow at their barbed ends? Do formins inhibit subunit addition or loss from filament ends (in common with capping proteins)? Can formins alone account for the initiation of formin-dependent actin filament bundles in yeast and other cells, or are other factors involved? The stage is now set for a mechanistic analysis of actin filament nucleation by formins.

THOMAS D. POLLARD

Department of Molecular, Cellular and Developmental Biology, Yale University

e-mail: thomas.pollard@yale.edu

\section{Chromosome instability: lessons from HIV}

Jan Paul Medema and René H. Medema

\begin{abstract}
Modulating the cell cycle is essential for viral replication and infection. HIV is an expert at manipulating these processes. New work now shows how apoptosis of CD4/CXCR4-expressing T cells during the course of HIV-infection is intimately connected to aberrant cell-cycle regulation.
\end{abstract}

$\mathrm{T}$ he cell-cycle machinery is carefully controlled by several checkpoints that monitor the proper order and fidelity of cell division. Modulating this machinery is essential for a productive viral infection, but is equally important when cells become cancerous. Over the years it has become increasingly clear that viruses are experts in tuning the cell-cycle apparatus to their own advantage. As such, studies of viral reproduction have not only resulted in vital information on viral life cycles but have also provided crucial information on the driving forces of the cell-cycle machinery and the regulation of its checkpoints.

A recent paper by Kroemer and colleagues provides another excellent example of such a dual hit ${ }^{1}$. It describes the mechanism that underlies HIV-induced apoptosis of CD4/CXCR4-expressing T cells, a process thought to be responsible for the T-cell depletion seen in HIV-infected individuals.
But the impact of their observations goes beyond HIV pathogenesis. The syncytial apoptosis studied in their paper constitutes a situation in which cells with supernumerary centrosomes attempt cell division. This process occurs in many cancerous cells, and is thought to contribute to the aneuploidy and genomic instability of transformed cells $^{2}$. Insight into the mechanisms responsible for the apoptotic demise of the syncytia may, therefore, help us to unravel the 